\title{
Efficient leukocyte depletion by a novel microfluidic platform enables the molecular detection and characterization of circulating tumor cells
}

\author{
Eva Obermayr ${ }^{1}$, Elisabeth Maritschnegg ${ }^{1}$, Christiane Agreiter ${ }^{1}$, Nina Pecha ${ }^{1,2}$, Paul \\ Speiser ${ }^{1,2}$, Samir Helmy-Bader ${ }^{2}$, Sabine Danzinger ${ }^{3}$, Michael Krainer ${ }^{4}$, Christian \\ Singer ${ }^{3}$ and Robert Zeillinger ${ }^{1}$ \\ ${ }^{1}$ Molecular Oncology Group, Department of Obstetrics and Gynecology, Comprehensive Cancer Center, Medical University of \\ Vienna, Waehringer Guertel 18-20, 1090 Vienna, Austria \\ ${ }^{2}$ Department of Obstetrics and Gynecology, Clinical Division for General Gynecology and Gynecological Oncology, \\ Comprehensive Cancer Center, Medical University of Vienna, Waehringer Guertel 18-20, 1090 Vienna, Austria \\ ${ }^{3}$ Department of Obstetrics and Gynecology, Division of Senology, Comprehensive Cancer Center, Medical University of \\ Vienna, Waehringer Guertel 18-20, 1090 Vienna, Austria \\ ${ }^{4}$ Department of Medicine I, Clinical Division of Oncology, Comprehensive Cancer Center, Medical University of Vienna, \\ Waehringer Guertel 18-20, 1090 Vienna, Austria
}

Correspondence to: Eva Obermayr, email: eva.obermayr@meduniwien.ac.at

Keywords: circulating tumor cells, microfluidics, gene expression analysis, ovarian cancer

Received: December 15, 2016

Accepted: September 30, 2017

Published: November 28, 2017

Copyright: Obermayr et al. This is an open-access article distributed under the terms of the Creative Commons Attribution License 3.0 (CC BY 3.0), which permits unrestricted use, distribution, and reproduction in any medium, provided the original author and source are credited.

\section{ABSTRACT}

RT-qPCR is a highly sensitive approach to detect rare transcripts, as derived from circulating tumor cells (CTCs) in the blood of cancer patients. However, the presence of unwanted leukocytes often leads to false positive results. Here, we evaluated whether the micro-fluidic Parsortix ${ }^{\mathrm{TM}}$ technology is appropriate to remove these leukocytes and thereby finally to improve the overall approach.

In this study, we established a workflow including the micro-fluidic Parsortix ${ }^{\mathrm{TM}}$ technology for the molecular detection of CTC related transcripts. Background levels of EPCAM, PPIC, TUSC3, and MAL2 were efficiently removed due to an up to $10^{6}$ fold depletion of leukocytes. The presence of these gene markers was observed in Parsortix ${ }^{\mathrm{TM}}$-enriched blood samples from patients with primary and recurrent gynecological cancer ( $32 \%$ and $14 \%$ ), as well as in $86 \%$ of the metastatic breast cancer samples, at a very high specificity. In the ovarian cancer samples, PPIC was the most prominent gene marker, contributing to all positive cases and at least to $70 \%$ of the positive cases after pre-amplification of the respective target genes. Expanding the analytical panel up to $\mathbf{2 9}$ gene markers further increased the positivity rate (primary gynecological cancer: $95 \%$, recurrent gynecological cancer: $100 \%$, metastatic breast cancer: $92 \%$ ).

The established workflow strongly improved the overall molecular analysis of the target cells by the efficient removal of contaminating cells, and, thereby offers great promise for the molecular characterization of CTCs.

\section{INTRODUCTION}

The analysis of circulating tumor cells (CTCs) in the blood of cancer patients represents an enormous technical challenge, not only due to their low absolute numbers but also the extreme abundance of blood cells. At early stages of cancer, CTC counts were reported to be as low as 1 to 5 cells in $7.5 \mathrm{ml}$ of blood [1], whereas in the metastatic setting, more than 1000 CTCs per $7.5 \mathrm{ml}$ blood have been observed [2].

Prior to analysis, the standard recommendation is to increase the relative amount of CTCs. Density gradient 
centrifugation is one approach to enrich the blood sample for CTCs: it is easy to perform, inexpensive, suitable for large blood volumes (i.e. $>10 \mathrm{ml}$ ), and will not - in contrast to immune-magnetic enrichment - favor a certain CTC subtype, e.g. those cells expressing the epithelial cell adhesion molecule EpCAM. However, density gradient centrifugation will reduce the number of leukocytes only to a certain extent (by 10- to 100-fold) [3]. A further depletion of unwanted cells can be achieved by immune-magnetic capture of white blood cells using an antibody against the leukocyte-specific CD45. At first glance, this approach may be tempting, but with large blood volumes it is associated with high costs, CTCs may be trapped within the bulk of captured leukocytes, or may even bind to the magnetic beads [4].

Over the past several years, we focused on the molecular analysis of CTCs using quantitative PCR (RTqPCR). Molecular assays for CTC detection offer many advantages, including the high sensitivity, small sample volumes, and the option to multiplex reactions and to analyze at high throughput; however, pre-analytical issues such as blood sample processing may be more crucial than with immune-fluorescent based assays [5]. In our earlier RT-qPCR-based studies we identified a 6-gene panel for the detection of CTCs in breast cancer and gynecological malignancies [6], and a further panel (comprising the cyclophilin $\mathrm{C}$ encoding gene PPIC) specifically for ovarian cancer [7]. In both studies, we used density gradient centrifugation to enrich the CTCs. The high amount of residual leukocytes in the enriched samples led to false positive results, most likely due to illegitimate transcription of the selected genes in leukocytes contaminating the enriched CTC sample. Although we were able to correlate the expression of the CTC-related molecular markers with patient outcome, we supposed that a better depletion of leukocytes would reduce the RT-qPCR background and improve both specificity and sensitivity of the molecular approach.

Further studies using molecular assays for the detection of CTC-related transcripts are primarily based on the immune-magnetic enrichment of the CTCs prior to PCR [8-12], which may achieve an appropriate depletion of leukocytes depending on the respective protocol. However, the presence of the targeted cell surface epitopes may be diminished on a subpopulation of CTCs displaying a more mesenchymal than an epithelial phenotype $[13,14]$. Several studies have recently shown that the portion of epithelial-like CTCs may be low in ovarian cancer [7, 15-17], suggesting that more comprehensive enrichment strategies based on physical and not on biological CTC properties alone may be more appropriate.

Recently, ANGLE plc launched the CE-marked Parsortix ${ }^{\mathrm{TM}}$ cell separation system for the research market. The FDA clearance process for the diagnostic market in the U.S. (www.angleplc.com) is underway. The Parsortix ${ }^{\mathrm{TM}}$ system uses a micro-fluidic technology to isolate rare cells (e.g. CTCs) based on their less deformable nature and usually larger size compared to blood cells. The separation of the blood components takes place in a microscope slide sized disposable cassette, which contains a series of steps leaving a $10 \mu \mathrm{m}$ measuring gap between the top cover and the final step. The captured cells may either be harvested for subsequent analysis, or alternatively stained within the cassette. The advantages of the technology are the antigenindependent enrichment of the target cells, the reported high purity of the captured cells, and flexibility in terms of blood volume to be processed. Thus we assumed that the Parsortix ${ }^{\mathrm{TM}}$ technology may be appropriate for the subsequent molecular analysis of the captured cells.

The aim of this study was to improve the molecular analysis of CTC-related markers by eliminating any background due to residual leukocytes. We developed a workflow which employed a density gradient preenrichment step, a final enrichment step using the Parsortix ${ }^{\mathrm{TM}}$ technology, and the RT-qPCR based analysis of 29 genes. The established protocol is flexible in terms of sample volume and number of gene markers analyzed, and thus offers great promise for further research on the molecular detection and characterization of CTCs in clinical studies.

\section{RESULTS}

\section{Optimization of the separation workflow}

First we evaluated whether the Parsortix ${ }^{\mathrm{TM}}$ technology and default separation conditions ( $2 \mathrm{ml}$ blood, 23 mbar pressure, $10 \mu \mathrm{m}$ final step size) can be applied for ovarian cancer cells. Despite their small size, TOV21G (median diameter $11 \mu \mathrm{m}$, IQR $8-10 \mu \mathrm{m}$ ) and CaOV3 (median diameter $10 \mu \mathrm{m}$, IQR $8-12 \mu \mathrm{m}$ ) cells were captured at mean rate of $28.4 \%$ and $71.8 \%$, respectively (see Figure 1A-1C). The number of captured tumor cells significantly increased with the number of tumor cells added to the blood sample (TOV21G: Pearson $r=0.998$, $p=0.045$; CaOV3: Pearson $\mathrm{r}=0.904, p=0.005$ ).

The number of captured tumor cells in a given patient sample and thus the overall sensitivity can only be increased by processing larger blood samples. To avoid long processing time, volumes larger than the default volume of $2 \mathrm{ml}$ can only be processed by two ways: either by an upfront pre-enrichment step in order to remove the majority of the blood cells (protocol A), or by increasing the pressure and thus the separation flow rate (protocol B). We observed that with both protocols the overall recovery rate was lower than at the default condition; however, due to the larger sample volume, the absolute number of captured tumor cells was higher (Figure 1C). Furthermore, we observed that the overall recovery rate of TOV2 $1 \mathrm{G}$ cells spiked into the blood was higher with protocol A than with B (mean $18.7 \%$ vs. $12.3 \%$ ), whereas 
with $\mathrm{CaOV} 3$ spiked samples it was exactly the opposite $(11.8 \%$ vs. $28 \%)$.

\section{Evaluation of the molecular analysis using spiked samples}

Next, we performed $C D 45$-specific RT-qPCR in order to evaluate whether the CD45 RNA expression levels can serve as an estimate for the leukocyte contamination. As expected, the $\lg 2$ transformed $\mathrm{Cq}$ values correlated with the amount of residual leukocytes in the enriched samples (Pearson $r=-0.918, p<0.001$, see Figure 2A). Furthermore, $C D 45$-specific RT-qPCR indicated that the number of residual leukocytes was significantly smaller in samples enriched by protocol A than in those which had been processed using protocol B (see Figure 2B; unpaired $t$ test with Welch's correction $p=0.002$ ). Thus we assumed that protocol A would be more appropriate for the subsequent molecular analysis.

Then, we analyzed the transcript levels of the selected CTC gene markers (MAL2, LAMB1, SERPINE2, $P P I C, T U S C 3$, and $E P C A M)$ in each three healthy donor blood samples processed using the default settings, protocol A, and $\mathrm{B}$. We did not observe an amplification signal specific for EpCAM, PPIC, MAL2, and TUSC3 in any of these samples. As LAMB1 and SERPINE2 transcripts were detected in all of the samples (see Figure 2C), these markers were excluded from further analyses.

Finally we added fluorescently labeled tumor cells at defined numbers to healthy donor blood, and processed these samples using the respective protocols. After the

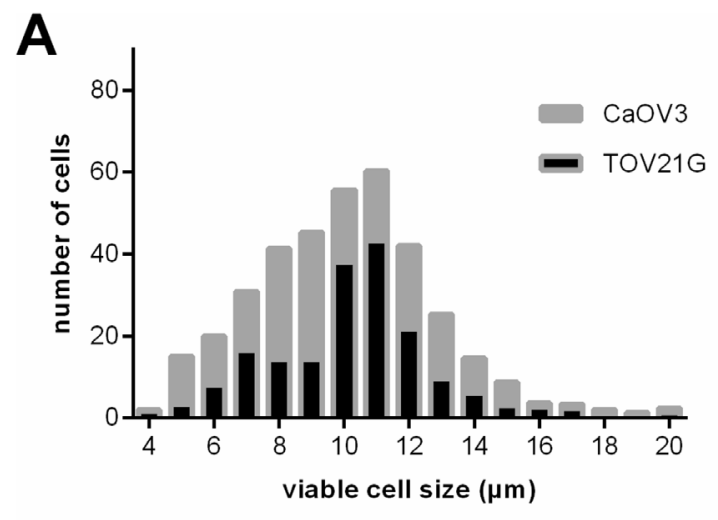

B
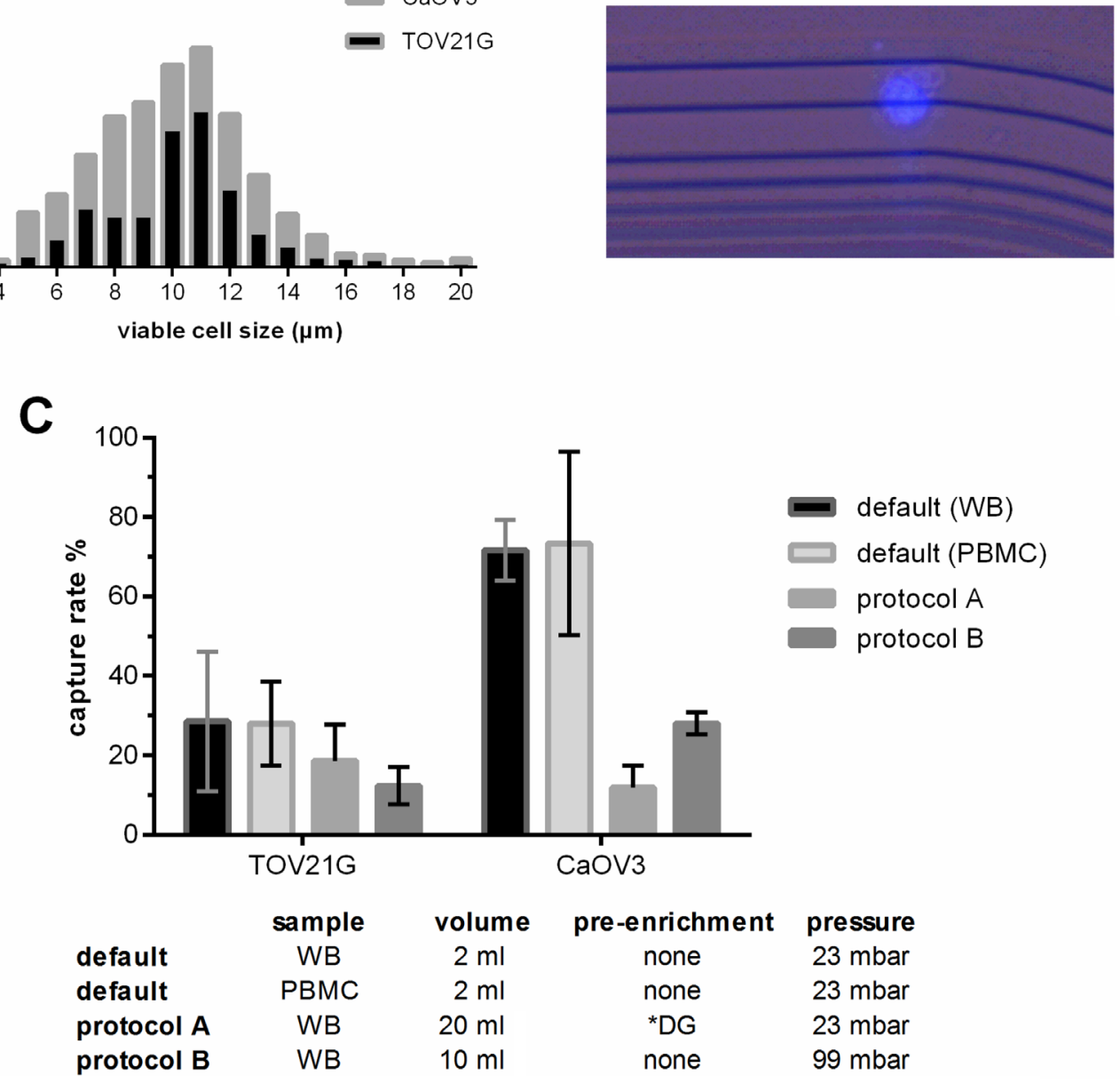

Figure 1: Characteristics of the applied protocols used for the enrichment of blood samples employing the Parsortix ${ }^{\mathrm{TM}}$ technology. (A) Size distribution of viable TOV21G and CaOV3 cells. The size of the viable cells was measured using the Countess ${ }^{\circledR}$ automated cell counter. (B) Combined fluorescence and bright field micrograph of a CellTrace Violet pre-labeled TOV21G cell captured on the separation structure in the Parsortix ${ }^{\mathrm{TM}}$ microfluidic cassette. (C) Capture rate of TOV21G and CaOV 3 tumor cells added to the sample at equal numbers (i.e. 5 cell per ml). To test the default settings, the tumor cells were added to whole blood (WB) and to a cell fraction (PBMC) after enrichment. To test protocols A and B, the tumor cells were added to whole blood. *DG density gradient centrifugation. 
enrichment and before lysis, the number of the tumor cells captured in the microfluidic cassette was assessed. Then RT-qPCR specific for EpCAM, PPIC, MAL2, and TUSC 3 was performed. The resulting $\mathrm{Cq}$ values correlated with the number of captured cells (Figure 3 ). Thus, in the samples spiked with TOV21G cells, the strongest amplification signals were observed with protocol $\mathrm{A}$, in samples which contained the highest absolute number of tumor cells due to the large sample volume (Figure 3A and $3 \mathrm{~B})$. The amplification signal was smaller in protocol
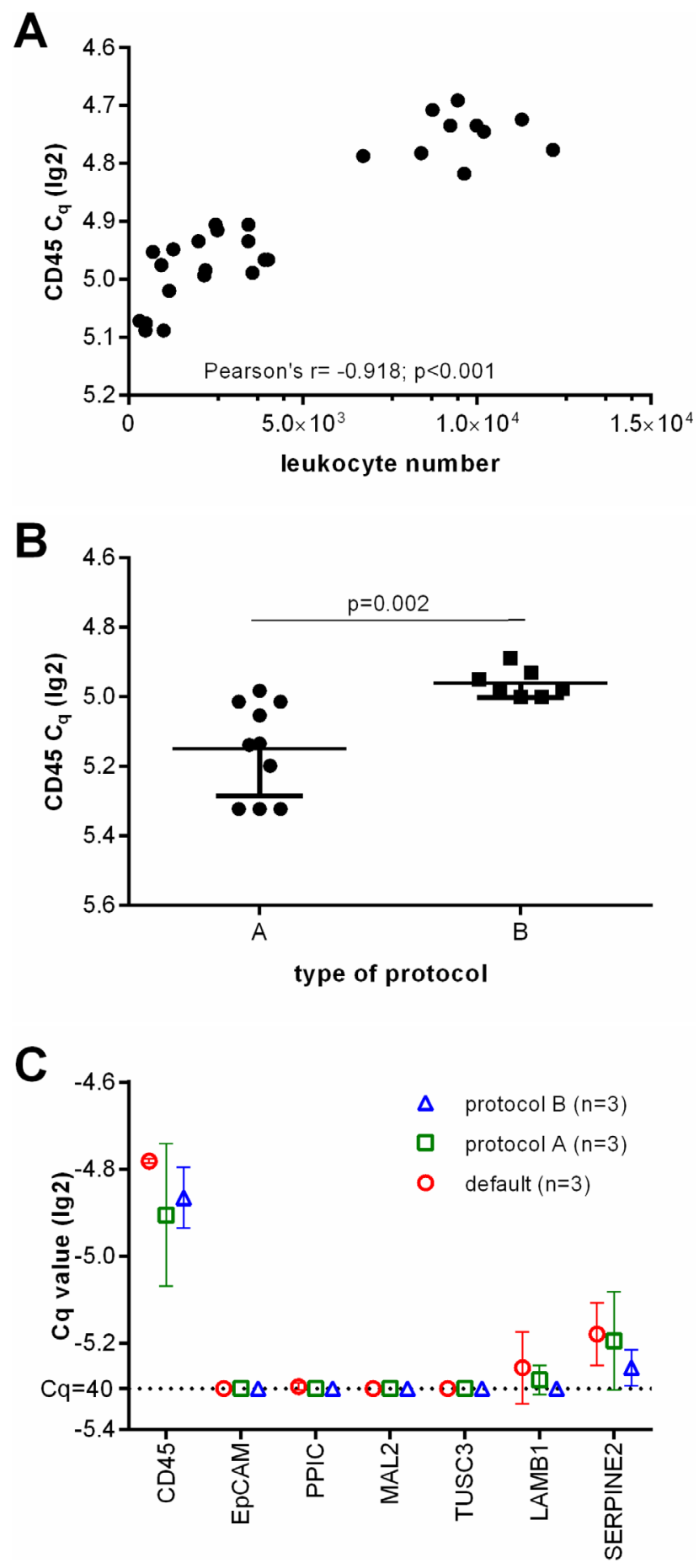

Figure 2: Depletion of leukocytes of the applied protocols. (A) Showing the correlation of leukocyte numbers with $\lg (2)$ transformed $\mathrm{Cq}$ values of CD45 gene expression as assessed by RT-qPCR. (B) $\lg (2)$ transformed Cq values of CD45 gene expression of healthy donor blood samples processed by protocol A and B. (C) Relative gene marker expression levels after Parsortix ${ }^{\mathrm{TM}}$ based separation. Each three healthy donor blood samples were processed using default settings starting with $2 \mathrm{ml}$ blood, protocol A starting with $20 \mathrm{ml}$ blood (with an upfront density gradient enrichment), or using protocol B starting with $10 \mathrm{ml}$ blood (applying an increased pressure of $99 \mathrm{mbar}$ ). 


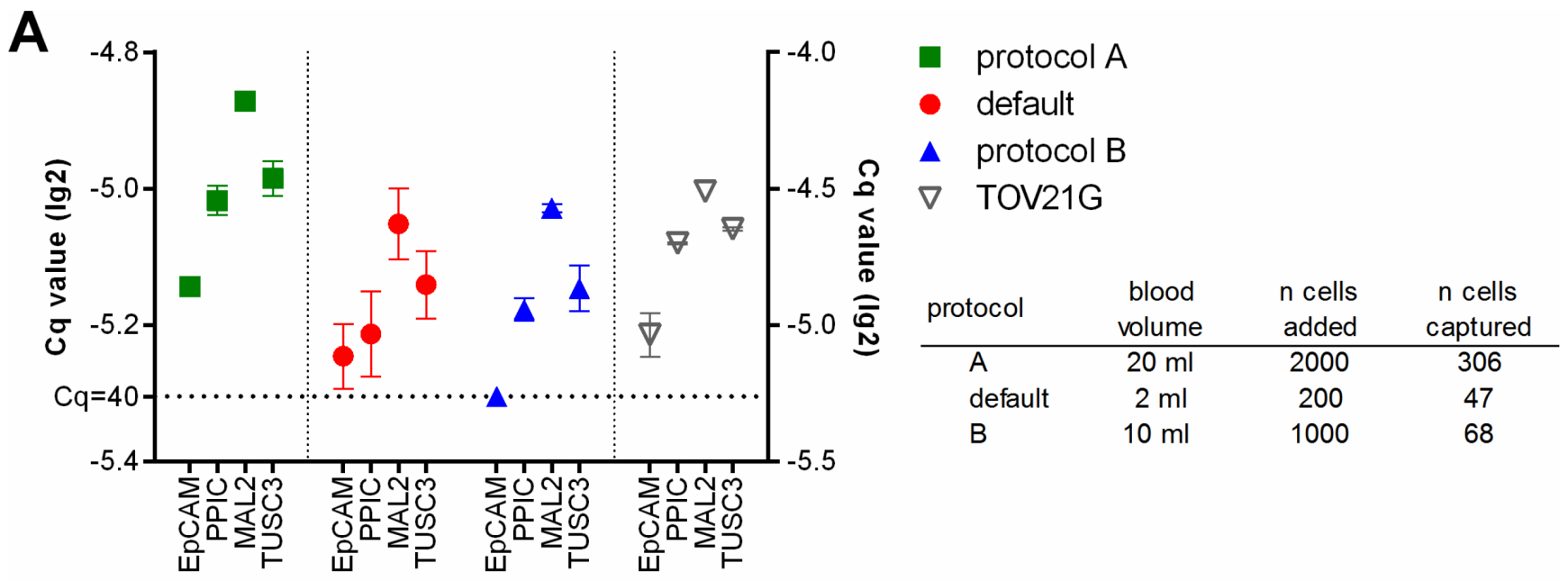

B
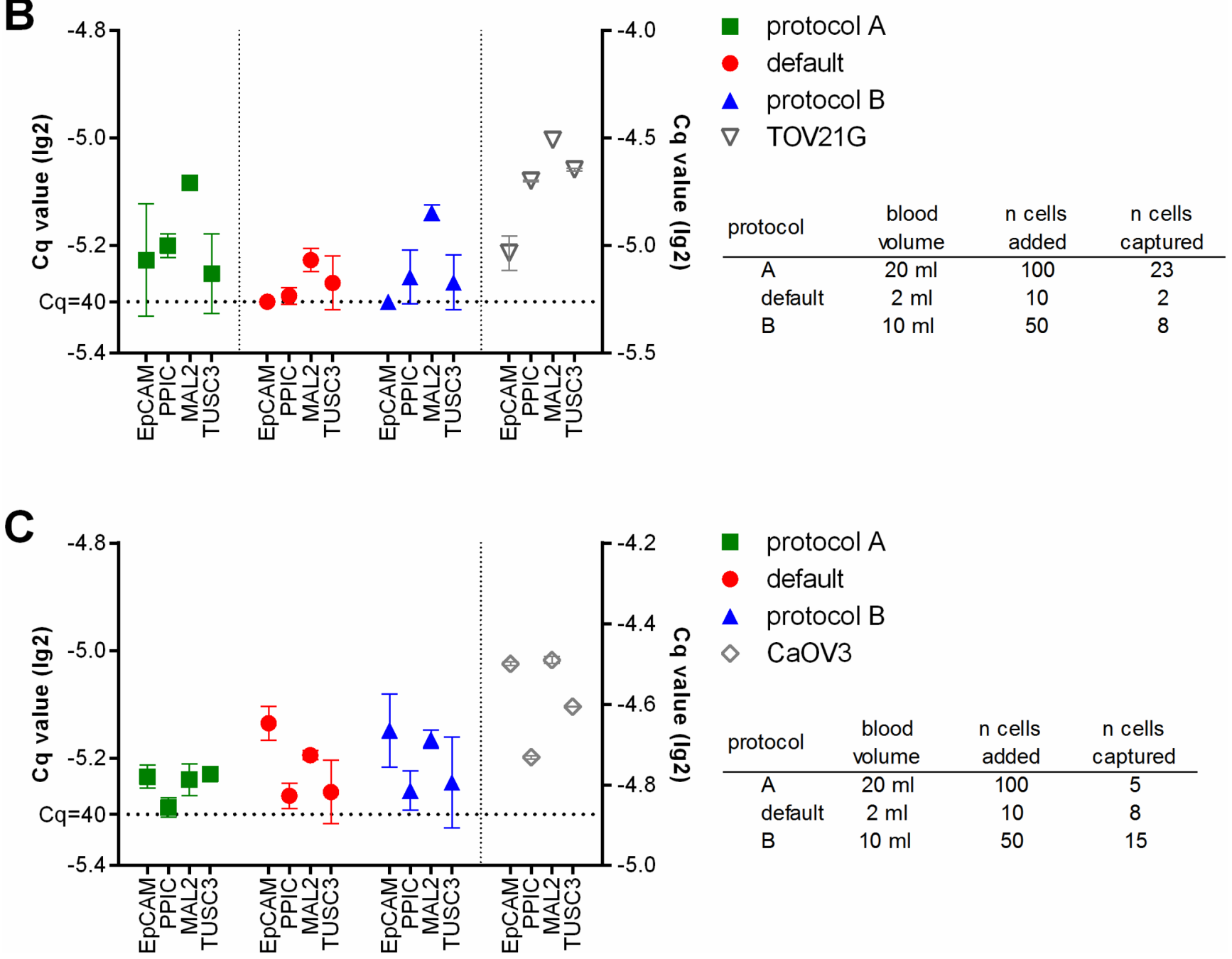

Figure 3: Relative gene expression levels in healthy donor blood samples spiked with TOV21G (A and B) or CaOV3 (C) tumor cells. The sample volume was determined by the respective protocol used (default: $2 \mathrm{ml}$, protocol A: $20 \mathrm{ml}$, protocol B: 10 $\mathrm{ml})$. The tumor cells were added to the samples at high $(\mathrm{A}: 100 \mathrm{cells} / \mathrm{ml})$ and low $(\mathrm{B}$ and $\mathrm{C}: 5 \mathrm{cells} / \mathrm{ml})$ concentrations. Then the samples were processed using the respective protocols. The RT-qPCR was done without prior pre-amplification of the targets. The resulting $\lg (2)$ transformed $\mathrm{Cq}$-values are plotted onto the left y-axis. RT-qPCR of the pure tumor cells was performed for comparison ( $\lg (2)$-transformed Cq-values plotted onto the right y-axis). 
B samples and those samples which had been processed at default settings. In contrast, the strongest amplification signals in $\mathrm{CaOV} 3$ spiked samples were measured in samples processed using protocol B (Figure 3C). Here, the absolute number of captured cells was higher in protocol B than in protocol A (see also Figure 1C), although initially a smaller number of tumor cells had been added to the sample. Possibly, a poor recovery rate of the density gradient enrichment led to the lower number of cells in the final sample.

\section{Molecular analysis of patients' blood samples, with and without pre-amplification}

In the next step, we processed blood samples from 36 cancer patients and 12 healthy donors (cohort 1) using protocol A and measured the gene expression levels of EPCAM, MAL2, PPIC, and TUSC3 in the enriched cell fractions. Twenty-two samples were taken at primary diagnosis before any therapeutic intervention (cervical cancer $N=3$, endometrial cancer $N=4$, ovarian cancer $\mathrm{N}$ $=13$, vulvar cancer $N=2$ ), whereas fourteen samples were taken from patients with recurrent disease (ovarian cancer $N=7$ ) and from patients with metastatic breast cancer $(N=7)$. The respective gene transcripts were detected in $7 / 22(32 \%)$ samples taken at primary diagnosis, in $1 / 7$ (14\%) samples at recurrence, in 6/7 (86\%) breast cancer samples, and in none of the 12 healthy donor samples (see Figure 4, left panel).

Then, we investigated the effects of a preamplification step on the measured transcript levels in the same samples. In the healthy donor samples, the transcript levels of PPIC, MAL2, and TUSC3 were still below the detection limit of RT-qPCR. An EpCAM specific amplification signal was observed in one single case. Among the same 36 cancer patients included in cohort 1, after a pre-amplification step at least one of the transcripts was observed in $12 / 22(55 \%)$ samples taken at primary diagnosis, in 5/7 (71\%) samples at recurrence, and in all (100\%) breast cancer samples (Figure 4, right panel).

\section{Expanding the molecular analysis by additional gene markers}

In a further set of 54 blood samples taken from 11 healthy female donors and from 43 cancer patients (cohort 2) we investigated the effect of additional CTCrelated gene markers to the analysis (see Supplementary Table 1). In addition to the four CTC-related markers analyzed before (PPIC, MAL2, TUSC3, and EpCAM), the gene expression of further 25 markers was measured using RT-qPCR (see Figure 5). From all 29 markers analyzed, two markers ( $C D H 3$ and $S C G B 2 A 2)$ were negative in in all samples (see Figure 5A). Nine markers (PRAME, EPCAM, TUSC3, GPX8, PPIC, AGR2, CDH2, TFF1, and $P G R$ ) were negative in all of the healthy control samples investigated. These nine markers contributed to RT-qPCR positive cases in $18 / 22(82 \%)$ samples taken at primary diagnosis, in $8 / 12(67 \%)$ breast cancer samples, and in $8 / 9$ $(89 \%)$ samples at recurrence (see Figure 5B).

The remaining 18 markers (see Figure 5C) were positive in the healthy control samples at various rates ranging from just $9 \%(L A M B 1,1 / 11)$ to $100 \%$ positive cases (VIM and ERCC1). However, we observed that the relative gene expression was higher in the cancer patients than in the healthy control samples. By choosing the two-fold standard deviation from the mean gene expression level in the control samples as threshold value, six from those nine cancer samples, which did not show any amplification signal related to $P G R, T F F 1, C D H 2$, AGR2, PPIC, GPX8, TUSC3, EPCAM, and PRAME were additionally assigned as being positive. Thus, the analysis of 29 markers resulted in a positivity rate of $95 \%$ in primary cancer, of $92 \%$ in breast cancer, and of $100 \%$ in recurrent cancer samples.

\section{DISCUSSION}

In this study we established a workflow combining density gradient centrifugation and enrichment by the Parsortix ${ }^{\mathrm{TM}}$ microfluidic technology, followed by RTqPCR analysis. The Parsortix ${ }^{\mathrm{TM}}$ technology alone or in combination with a density gradient enrichment has mainly been used for subsequent immuno-staining and single cell analysis so far [18-21]. Our workflow meets the key prerequisite for RT-qPCR based analysis of CTC-related gene markers in the obtained cell sample by efficiently removing unwanted leukocytes, which could contribute to false positives due to illegitimate transcription of the used markers. Furthermore, the protocol can be adapted to large sample volumes in order to counteract the low abundance of target cells, especially in early stage disease or in cancer types which may not primarily spread via the hematogenous route like ovarian cancer.

By combining density gradient centrifugation and the Parsortix ${ }^{\mathrm{TM}}$ separation, one third of the blood samples obtained from patients with primary ovarian cancer (cohort 1) were RT-qPCR-positive prior to pre-amplification. Of note, $P P I C$ was the only gene marker which was detected in these samples. These results are in line with those obtained from our earlier study employing density gradient centrifugation alone, yielding 24\% RT-qPCR-positive cases in primary ovarian cancer, mostly attributed to PPIC-positivity [7]. Noteworthy, in the same set of cohort 1 samples the percentage of RT-qPCR positive cases had more than doubled by adding a pre-amplification step prior to RT-qPCR. After pre-amplification, PPIC still contributed to $78 \%$ of the positive ovarian cancer samples in cohort 1 , and to $70 \%$ in cohort 2 .

Whether the observed high frequency of investigated gene markers is indeed related to the presence of CTCs or rather to other cells, like circulating endothelial cells [22] 


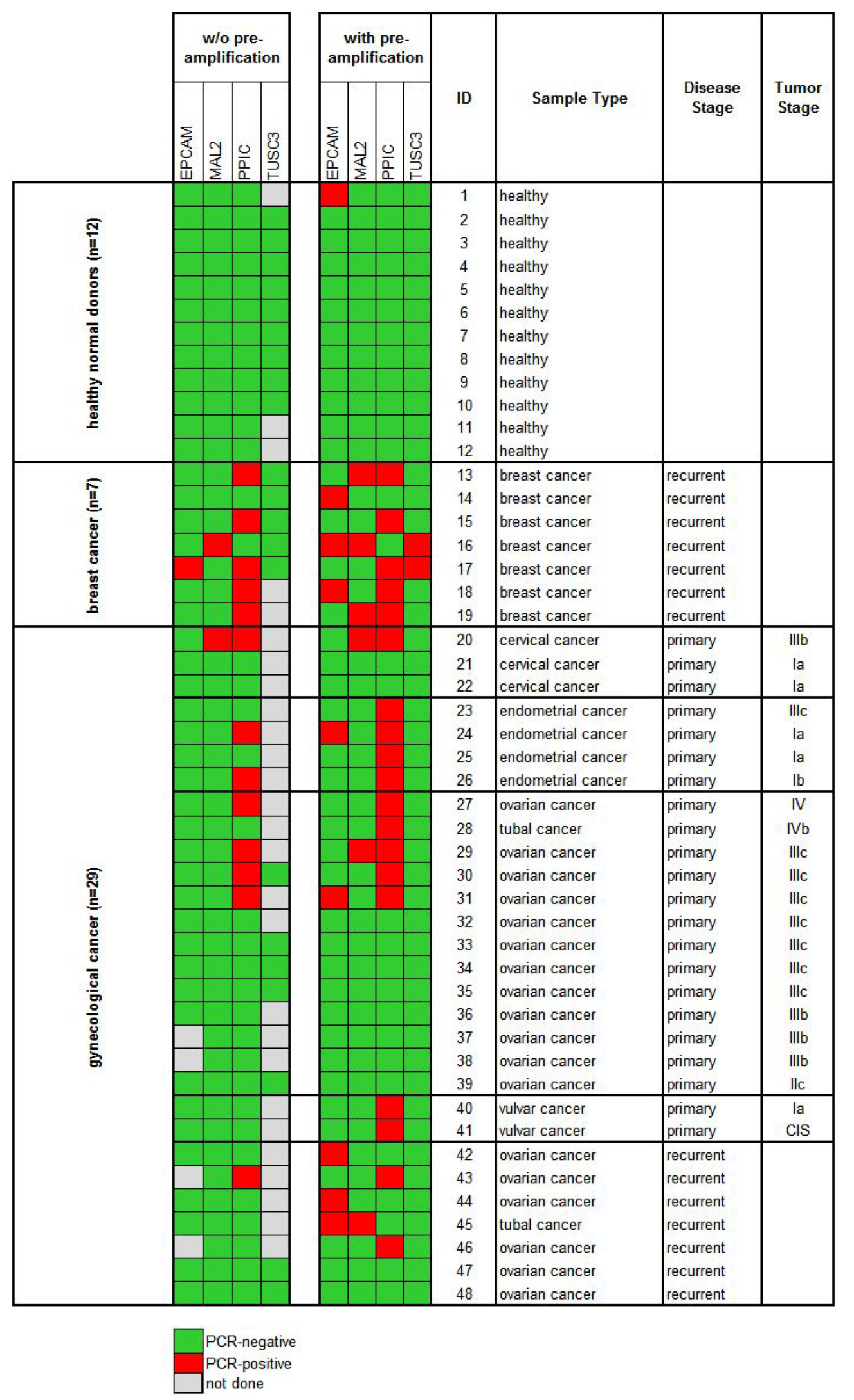

Figure 4: Heat map of EPCAM, MAL2, PPIC, and TUSC3 gene expression analysis of Parsortix ${ }^{\mathrm{TM}}$ enriched blood samples (cohort 1). Each $20 \mathrm{ml}$ blood from 12 healthy donors and 36 cancer patients were processed using protocol A (comprising an upfront density gradient enrichment). RT-qPCR was performed without (left panel) and with prior pre-amplification (right panel) of the respective transcripts. Tumor stages of the primary gynecological cancers are given according to the FIGO classification system. 
or cells of hematogenous origin still needs to be clarified in future studies [23]. However, our earlier study on the molecular characterization of CTCs in ovarian cancer had already shown the abundance and prognostic relevance of PPIC transcripts in density gradient enriched cell fractions [7]: PPIC was identified among other genes as putative
CTC marker by gene expression analysis of paired tumor tissue and PBMC samples. The present results indicate that the high sample purity as provided by the Parsortix ${ }^{\mathrm{TM}}$ technology renders the possibility to expand the molecular analysis of the captured cells by employing a preamplification step for up to 100 different template species,
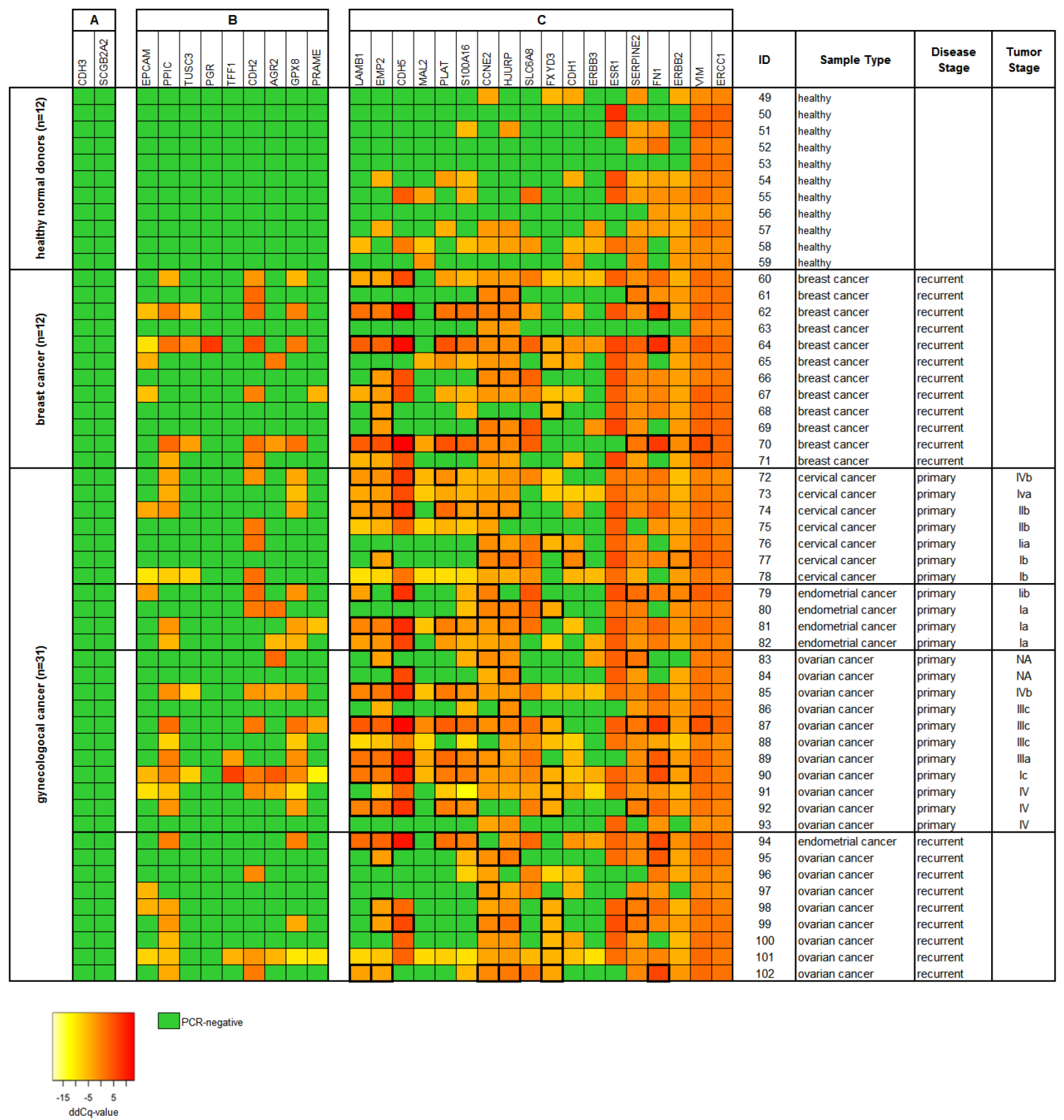

Figure 5: Heat map of relative gene expression levels of 29 CTC-related gene markers of Parsortix ${ }^{\mathrm{TM}}$ enriched blood samples (cohort 2). Each $20 \mathrm{ml}$ blood from 11 healthy donors and 43 cancer patients were processed using protocol A (comprising an upfront density gradient enrichment). RT-qPCR was performed following a pre-amplification step of the respective transcripts. Raw Cq values were normalized to the reference gene $B 2 M$, resulting in $\mathrm{dCq}$ values. ddCq values were obtained by normalizing $\mathrm{dCq}$ values to a calibrator sample. The gene markers are ranked according to their respective gene expression levels in the samples from (A) undetected in both healthy donors and cancer patients, (B) undetected in healthy donors but not in cancer patients, and (C) detected in both healthy donors and cancer patients. ddCq levels above the two-fold standard deviation from the mean gene expression in the healthy donor samples are framed with thick black lines. Tumor stages of the primary gynecological cancers are given according to the FIGO classification system. 
and consequently to increase the chance of identifying cells displaying a deviating phenotype [24]. Thus, our study may facilitate the molecular characterization of CTCs, and help to identify further prognostic or predictive gene markers.

The amount of residual leukocytes may be similar to that of immune-magnetic enrichment employing antibodies attached to magnetic beads; however, our approach is more flexible in terms of independency from cell surface markers as enrichment targets and availability of appropriate antibodies. To the best of our knowledge, our study is the first to demonstrate the feasibility of gene expression analysis in combination with Parsortix ${ }^{\mathrm{TM}}$ for the detection of CTC-related transcripts in cancer patients. In contrast to Gorges et al. who performed single cell analysis of Parsortix ${ }^{\mathrm{TM}}$ enriched cells [20], we show that the high depletion of leukocytes provided by our approach enables the detection of CTC-related transcripts in the total amount of harvested cells, which had only been demonstrated in blood samples spiked with tumor cells but not in patient samples so far [19].

Compared with an earlier study evaluating the use of the Parsortix ${ }^{\mathrm{TM}}$ system in prostate cancer [18], the capture rate of spiked tumor cells was lower in our study using ovarian cancer cell lines. The main reason probably is the smaller size of ovarian cancer cells, which will pass the Parsortix ${ }^{\mathrm{TM}}$ separation cassette more likely than larger cells like prostate and breast cancer cells. We used two quite different cell lines for the spiking experiments, in terms of EpCAM protein expression, morphology, mutational status, doubling time, et cetera [25]. In addition to these biological characteristics, their respective size may at least in part contribute to the different behavior in the pre-enrichment strategies involved, which are based on the physical properties of the cells, like size, deformability, or density: Starting from whole blood, $\mathrm{CaOV} 3$ cells were captured more efficiently than TOV21G cells. This difference may be explained by the wider size distribution of $\mathrm{CaOV} 3$ cells than of TOV21G cells. In contrast, after density gradient centrifugation, the final recovery of tumor cells was worse with $\mathrm{CaOV} 3$ cells. Parallel spiking experiments of $\mathrm{CaOV} 3$ cells into whole blood before density gradient centrifugation and into a PBMC fraction obtained after centrifugation yielding similar recovery rates (see Figure 1C) indicate that the density gradient centrifugation led to the major part of $\mathrm{CaOV} 3$ cell loss in protocol A. However, the pattern of gene expression in the microfluidic enriched tumor cells was similar to that of the same tumor cells before enrichment (see Figure 3 ), suggesting that the Parsortix ${ }^{\mathrm{TM}}$ separation may not be selective for a particular cellular subtype.

We measured the gene expression levels of $C D 45$ as an estimate for leukocyte contamination. The observed difference in CD45 levels between protocols $\mathrm{A}$ and $\mathrm{B}$ were in line with the total average amount of residual leukocytes, as specified by the manufacturer (i.e. 1300 vs. 3000 cells, personal communication with K. Mumford, ANGLE plc, January 2016). In concordance with another study evaluating the Parsortix ${ }^{\mathrm{TM}}$ system, the number of residual leukocytes did not depend on the sample volume, but rather on individual donors [21]. We observed that both the recovery of target cells and depletion of leukocytes were more variable in protocol A than in B. This phenomenon may be explained by varying performance either of the density gradient centrifugation or of the Parsortix ${ }^{\mathrm{TM}}$ separation at 23 mbar (protocol A), which was more prone to instability than at 99 mbar (protocol B), or of both. A weakness of our study design is that we did not compare both protocol A and B in the same patient samples; the main reason for this was the limited amount of blood, which was not sufficient for two microfluidic separations in parallel.

Based on our previous experience, our focus in the present study was on the best possible depletion of leukocytes. The significantly lower amount or leukocytes in protocol A enriched samples as compared to the ones enriched by protocol B was thus the main reason to choose protocol A for processing real patient samples. Being aware of the low yield of captured tumor cells, we are going to test further protocols for our application, in order to increase the performance of the Parsortix ${ }^{\mathrm{TM}}$ separation and the overall capture rate of the target cells. For these purposes, the density gradient centrifugation step could be replaced, e.g. by a second microfluidic separation step (as described in [21]), or separation cassettes with a smaller than $10 \mu \mathrm{m}$ measuring critical step size could be evaluated to find the optimum balance between capture rate and sample purity. Indeed, since starting our study, the manufacturer has developed alternative cassettes with $6.5 \mu \mathrm{m}$ and $8 \mu \mathrm{m}$ critical step sizes, which should improve CTC capture in principle.

In conclusion, we have developed a workflow for the enrichment of CTCs, which assures the depletion of contaminating leukocytes up to $10^{6}$-fold and thus allows for the subsequent molecular analysis by providing a high purity of the enriched cells. Our protocol offers great promise for further research on the molecular detection and characterization of CTCs in clinical studies. Future efforts include further improving the recovery rate of the CTCs, validating the overall approach in larger sample cohorts, and evaluating the prognostic value of further CTC gene markers besides the PPIC gene.

\section{MATERIALS AND METHODS}

\section{Blood samples}

Blood samples were taken from patients suffering from gynecological malignancies, including ovarian, endometrial, and cervical cancer, and from patients with metastatic breast cancer at the General Hospital 
in Vienna. Control blood samples were acquired from female healthy donors without a history of cancer. All samples were taken before any therapeutic intervention after written informed consent. The blood was collected in Vacuette EDTA tubes (Greiner Bio-One) and processed within four hours. The study was approved by the Ethic Committee of the Medical University of Vienna, Austria (EK366/2003).

\section{Cell spiking experiments}

The ovarian cancer cell lines TOV21G and CaOV3 were trypsinized at about $70 \%$ confluence and incubated with the fluorescent CellTrace Violet stain (Invitrogen) according to the manufacturer's protocol. The mean cell diameter was measured using the Countess ${ }^{\circledR}$ automated cell counter (Invitrogen). A healthy female donor blood sample was spiked with the fluorescently labeled cells by transferring single tumor cells with a pipette until a final concentration of 5 and 100 tumor cells per ml blood was achieved. The blood sample volume was $2 \mathrm{ml}, 20 \mathrm{ml}$ and $10 \mathrm{ml}$, in order to test the separation at default conditions, with protocol A and protocol B, respectively. Un-spiked blood samples from female healthy donors were used as controls. The tumor cells captured within the Parsortix ${ }^{\mathrm{TM}}$ separation cassette were counted using a fluorescence microscope to assess the capture rate (absolute number of captured tumor cells divided by total number of added tumor cells).

\section{Processing of blood samples}

The blood samples were processed in three different ways: (i) Using the Parsortix ${ }^{\mathrm{TM}}$ default settings, $2 \mathrm{ml}$ blood was processed by applying a 23 mbar pressure to push the sample through the separation cassette. For processing larger blood volumes, either protocol A or protocol B was used. (ii) With protocol A, $20 \mathrm{ml}$ blood was pre-enriched by density gradient centrifugation using $15 \mathrm{ml}$ Percoll (GE Healthcare; $d=1.065 \mathrm{~g} / \mathrm{ml}, 305 \mathrm{mOsm} / \mathrm{kg}$ ) in Sepmate ${ }^{\circledR}$ tubes (Stemcell Technologies). After centrifugation at $4^{\circ} \mathrm{C}$ at $1350 \mathrm{x}$ g for 20 mins with disabled brake, the enriched cells in the top layer were washed with PBS and further processed using the Parsortix ${ }^{\mathrm{TM}}$ technology by applying a 23 mbar pressure. (iii) With protocol B, the $10 \mathrm{ml}$ whole blood sample (diluted with $10 \mathrm{ml}$ PBS) was directly processed without any further pre-enrichment at a 99 mbar pressure. With each of the three protocols, Parsortix ${ }^{\mathrm{TM}}$ separation cassettes with a $10 \mu \mathrm{m}$ gap size were used. After separation the cassette was either visually examined under a microscope to count fluorescently labeled tumor cells (spiking experiments), and/or was flushed with 350 $\mu 1$ RLT lysis buffer (Qiagen) to retrieve the enriched cells for subsequent molecular analysis. The cell lysates were stored at $-20^{\circ} \mathrm{C}$ until RNA extraction.

\section{Selection of target genes}

The RT-qPCR markers were selected based on our earlier studies which compared the gene expression of healthy control blood samples and tumor cell lines [6], and of paired tumor tissue and PBMC samples using microarrays [7]. Both studies aimed at identifiying transcripts which may indicate the presence of CTCs in patient blood samples. In that former studies we validated 384 differentially expressed genes with RTqPCR. Here, we selected just a few of the genes most abundantly expressed in the ovarian cancer cell lines CaOV3 and TOV21G, which were used for the spiking experiments. In addition to these gene markers (MAL2, LAMB1, SERPINE2, PPIC, and TUSC3), we selected EpCAM as known marker specific for epithelial cells, $C D 45$ as a marker for residual leukocyte content, and $\beta$-2-microglobulin $(B 2 M)$ as reference gene. In those experiments with an upfront pre-amplification step (which allows the analysis of up to 100 transcripts) we included further gene markers which had been investigated in our earlier studies and which had been previously described as being specific for epithelial or mesenchymal cells (Supplementary Table 1).

\section{Molecular analysis}

Total RNA was extracted from the cell lysates using the RNeasy Micro Kit (Qiagen) without DNase treatment. The total RNA amount was incubated with random nonamers (Sigma-Aldrich) at $70^{\circ} \mathrm{C}$ for 5 mins. After cooling to $4^{\circ} \mathrm{C}$, M-MLV Reverse Transcriptase, RNase H Minus, Point Mutant (Promega), reaction buffer, and RNAsin (Promega) were added. The $20 \mu 1$ reaction mix was incubated at $25^{\circ} \mathrm{C}$ for 15 mins, then at $45^{\circ} \mathrm{C} 50$ mins and at $55^{\circ} \mathrm{C} 10$ mins. Pre-amplification was performed using the same TaqMan ${ }^{\circledR}$ assays as with RT-qPCR and the TaqMan ${ }^{\circledR}$ PreAmp Mastermix according to the manual (Life Technologies). The uniformity of the pre-amplification of the target genes relative to $C D K N 1 B$ as reference gene was assessed according to the manual. RT-qPCR was done in duplicates in a $10 \mu 1$ total reaction volume on the ViiA7 Real-Time PCR System using exon spanning TaqMan ${ }^{\circledR}$ assays and TaqMan $^{\circledR}$ Universal Mastermix II (Life Technologies) with default thermal cycling parameters $\left(50^{\circ} \mathrm{C} 2\right.$ mins; $95^{\circ} \mathrm{C} 10$ mins; 40 cycles at $95^{\circ} \mathrm{C} 15 \mathrm{~s}$, $60^{\circ} \mathrm{C} 1 \mathrm{~min}$ ). Raw data were analyzed using the ViiA7 Software v1.1 with automatic threshold setting and baseline correction. $\mathrm{dCq}$ value were calculated by normalizing the mean $\mathrm{Cq}$ value of gene $\mathrm{X}$ to the mean $\mathrm{Cq}$ of $B 2 M$. $\mathrm{ddCq}$ values were calculated by normalizing dCqs to a reference sample. If an amplification signal was observed in the healthy donor samples, a cut-off threshold value was calculated by adding the twofold standard deviation of the mean observed ddCq value in the control samples [26]. A 
patient sample was then assigned positive if the ddCq value of the respective gene marker was beyond that threshold.

\section{Statistics}

Graphs and statistics were done using GraphPad Prism version 6.01 .

\section{Abbreviations}

CTC: circulating tumor cell; $\mathrm{Cq}$ : quantitation cycle; CD45: cluster of differentiation 45; EpCAM: epithelial cell adhesion molecule; LAMB1: laminin; beta 1; MAL2: mal; T-cell differentiation protein 2 (gene/pseudogene); PBMC: peripheral blood mononuclear cells; PPIC: peptidylprolyl isomerase C (cyclophilin C); RT-qPCR: reverse transcription quantitative polymerase chain reaction; SERPINE2: Serpin family E member 2; TUSC3 tumor suppressor candidate 3 .

\section{Author contributions}

EO and EM performed the analyses, interpreted the data, and wrote the manuscript; CA processed the blood samples and assisted with data preparation, SHB, NP, PS, SH, and SD contributed to data acquisition; MK, CS and RD designed the study and revised the manuscript. All authors approved the final version of the manuscript.

\section{ACKNOWLEDGMENTS}

The authors thank Eva Schuster and Barbara Holzer (Molecular Oncology Group) for their assistance in sample preparation, Angelika Geroldinger (Center for Medical Statistics, Informatics and Intelligent Systems, Medical University of Vienna) for providing support in data processing, the team at ANGLE plc for technical support, and all women who donated blood samples.

\section{CONFLICTS OF INTEREST}

The authors declare no conflicts of interest.

\section{FUNDING}

This study received support from ANGLE plc given in form of Parsortix ${ }^{\mathrm{TM}}$ devices and microfluidic separation cassettes.

\section{REFERENCES}

1. Pantel K, Alix-Panabières C, Riethdorf S. Cancer micrometastases. Nat Rev Clin Oncol. 2009; 6:339-51. https://doi.org/10.1038/nrclinonc.2009.44.
2. Cristofanilli M, Budd GT, Ellis MJ, Stopeck A, Matera J, Miller MC, Reuben JM, Doyle GV, Allard WJ, Terstappen LW, Hayes DF. Circulating tumor cells, disease progression, and survival in metastatic breast cancer. N Engl J Med. 2004; 351:781-91. https://doi.org/10.1056/NEJMoa040766.

3. Rosenberg R, Gertler R, Friederichs J, Fuehrer K, Dahm M, Phelps R, Thorban S, Nekarda H, Siewert JR. Comparison of two density gradient centrifugation systems for the enrichment of disseminated tumor cells in blood. Cytometry. 2002; 49:150-58. https://doi.org/10.1002/ cyto. 10161.

4. Stott SL, Hsu CH, Tsukrov DI, Yu M, Miyamoto DT, Waltman BA, Rothenberg SM, Shah AM, Smas ME, Korir GK, Floyd FP Jr, Gilman AJ, Lord JB, et al. Isolation of circulating tumor cells using a microvortex-generating herringbone-chip. Proc Natl Acad Sci USA. 2010; 107:18392-97. https://doi.org/10.1073/pnas.1012539107.

5. Lianidou ES. Gene expression profiling and DNA methylation analyses of CTCs. Mol Oncol. 2016; 10:43142. https://doi.org/10.1016/j.molonc.2016.01.011.

6. Obermayr E, Sanchez-Cabo F, Tea MK, Singer CF, Krainer M, Fischer MB, Sehouli J, Reinthaller A, Horvat R, Heinze G, Tong D, Zeillinger R. Assessment of a six gene panel for the molecular detection of circulating tumor cells in the blood of female cancer patients. BMC Cancer. 2010; 10:666. https://doi.org/10.1186/1471-2407-10-666.

7. Obermayr E, Castillo-Tong DC, Pils D, Speiser P, Braicu I, Van Gorp T, Mahner S, Sehouli J, Vergote I, Zeillinger R. Molecular characterization of circulating tumor cells in patients with ovarian cancer improves their prognostic significance -- a study of the OVCAD consortium. Gynecol Oncol. 2013; 128:15-21. https://doi.org/10.1016/j. ygyno.2012.09.021.

8. Tewes M, Aktas B, Welt A, Mueller S, Hauch S, Kimmig R, Kasimir-Bauer S. Molecular profiling and predictive value of circulating tumor cells in patients with metastatic breast cancer: an option for monitoring response to breast cancer related therapies. Breast Cancer Res Treat. 2009; 115:58190. https://doi.org/10.1007/s10549-008-0143-x.

9. Aktas B, Kasimir-Bauer S, Heubner M, Kimmig R, Wimberger P. Molecular profiling and prognostic relevance of circulating tumor cells in the blood of ovarian cancer patients at primary diagnosis and after platinum-based chemotherapy. Int J Gynecol Cancer. 2011; 21:822-30. https://doi.org/10.1097/IGC.0b013e318216cb91.

10. Aktas B, Müller V, Tewes M, Zeitz J, Kasimir-Bauer S, Loehberg CR, Rack B, Schneeweiss A, Fehm T. Comparison of estrogen and progesterone receptor status of circulating tumor cells and the primary tumor in metastatic breast cancer patients. Gynecol Oncol. 2011; 122:356-60. https://doi.org/10.1016/j.ygyno.2011.04.039.

11. Todenhöfer T, Hennenlotter J, Feyerabend S, Aufderklamm S, Mischinger J, Kühs U, Gerber V, Fetisch J, Schilling D, Hauch S, Stenzl A, Schwentner C. Preliminary experience on the use of the Adnatest ${ }^{\circledR}$ system for detection of 
circulating tumor cells in prostate cancer patients. Anticancer Res. 2012; 32:3507-13.

12. Raimondi C, Nicolazzo C, Gradilone A, Giannini G, De Falco E, Chimenti I, Varriale E, Hauch S, Plappert L, Cortesi E, Gazzaniga P. Circulating tumor cells: exploring intratumor heterogeneity of colorectal cancer. Cancer Biol Ther. 2014; 15:496-503. https://doi.org/10.4161/cbt.28020.

13. Bednarz-Knoll N, Alix-Panabières C, Pantel K. Plasticity of disseminating cancer cells in patients with epithelial malignancies. Cancer Metastasis Rev. 2012; 31:673-87. https://doi.org/10.1007/s10555-012-9370-z.

14. Königsberg R, Obermayr E, Bises G, Pfeiler G, Gneist M, Wrba F, de Santis M, Zeillinger R, Hudec M, Dittrich C. Detection of EpCAM positive and negative circulating tumor cells in metastatic breast cancer patients. Acta Oncol. 2011; 50:700-10. https://doi.org/10.3109/028418 6X.2010.549151.

15. Blassl C, Kuhlmann JD, Webers A, Wimberger P, Fehm T, Neubauer H. Gene expression profiling of single circulating tumor cells in ovarian cancer - Establishment of a multimarker gene panel. Mol Oncol. 2016; 10:1030-42. https:// doi.org/10.1016/j.molonc.2016.04.002.

16. Aktas B, Kasimir-Bauer S, Heubner M, Kimmig R, Wimberger P. Molecular profiling and prognostic relevance of circulating tumor cells in the blood of ovarian cancer patients at primary diagnosis and after platinum-based chemotherapy. Int J Gynecol Cancer. 2011; 21:822-30. https://doi.org/10.1097/IGC.0b013e318216cb91.

17. Chebouti I, Kasimir-Bauer S, Buderath P, Wimberger P, Hauch S, Kimmig R, Kuhlmann JD. EMT-like circulating tumor cells in ovarian cancer patients are enriched by platinum-based chemotherapy. Oncotarget. 2017; 8:48820 31. https://doi.org/10.18632/oncotarget.16179.

18. Xu L, Mao X, Imrali A, Syed F, Mutsvangwa K, Berney D, Cathcart P, Hines J, Shamash J, Lu YJ. Optimization and Evaluation of a Novel Size Based Circulating Tumor Cell Isolation System. PLoS One. 2015; 10:e0138032. https:// doi.org/10.1371/journal.pone.0138032.

19. Hvichia GE, Parveen Z, Wagner C, Janning M, Quidde J, Stein A, Müller V, Loges S, Neves RP, Stoecklein NH, Wikman H, Riethdorf S, Pantel K, Gorges TM. A novel microfluidic platform for size and deformability based separation and the subsequent molecular characterization of viable circulating tumor cells. Int J Cancer. 2016; 138:2894-904. https://doi.org/10.1002/ijc.30007.

20. Gorges TM, Kuske A, Röck K, Mauermann O, Müller V, Peine S, Verpoort K, Novosadova V, Kubista M, Riethdorf S, Pantel K. Accession of Tumor Heterogeneity by Multiplex Transcriptome Profiling of Single Circulating Tumor Cells. Clin Chem. 2016; 62:1504-15. https://doi. org/10.1373/clinchem.2016.260299.

21. Chudziak J, Burt DJ, Mohan S, Rothwell DG, Mesquita B, Antonello J, Dalby S, Ayub M, Priest L, Carter L, Krebs MG, Blackhall F, Dive C, Brady G. Clinical evaluation of a novel microfluidic device for epitope-independent enrichment of circulating tumour cells in patients with small cell lung cancer. Analyst (Lond). 2016; 141:669-78. https:// doi.org/10.1039/C5AN02156A.

22. Erdbruegger U, Dhaygude A, Haubitz M, Woywodt A. Circulating endothelial cells: markers and mediators of vascular damage. Curr Stem Cell Res Ther. 2010; 5:294302. https://doi.org/10.2174/157488810793351721.

23. Attard G, Kaye SB. Identifying prognostic signatures in the blood of ovarian cancer patients. Gynecol Oncol. 2013; 128:1-2. https://doi.org/10.1016/j.ygyno.2012.11.011.

24. Hayes DF, Paoletti C. Circulating tumour cells: insights into tumour heterogeneity. J Intern Med. 2013; 274:137-43. https://doi.org/10.1111/joim.12047.

25. Beaufort CM, Helmijr JC, Piskorz AM, Hoogstraat M, Ruigrok-Ritstier K, Besselink N, Murtaza M, van IJcken WF, Heine AA, Smid M, Koudijs MJ, Brenton JD, Berns EM, Helleman J. Ovarian cancer cell line panel (OCCP): clinical importance of in vitro morphological subtypes. PLoS One. 2014; 9:e103988. https://doi.org/10.1371/ journal.pone.0103988.

26. Mikhitarian K, Martin RH, Ruppel MB, Gillanders WE, Hoda R, Schutte H, Callahan K, Mitas M, Cole DJ. Detection of mammaglobin mRNA in peripheral blood is associated with high grade breast cancer: interim results of a prospective cohort study. BMC Cancer. 2008; 8:55. https:// doi.org/10.1186/1471-2407-8-55. 